for congenital heart disease. J Thorac Cardiovasc Surg 2002;123(1):110-8.

5. Dawson AL, Cassell CH, Riehle-Colarusso T, Grosse SD, et al. Factors associated withlate detection of critical congenital heart disease in newborns. Pediatrics 2013;132(3):e604-11.

6. Chang RK, Gurvitz M, Rodriguez S. Missed diagnosis of critical congenital heart disease. Arch Pediatr Adolesc Med 2008;162(10):969-74.

7. Fuchs IB, Müller H, Abdul-Khaliq H, Harder T, et al. Immediate and long-term outcomes in children with prenatal diagnosis of selected isolated congenital heart defects. Ultrasound Obstet Gynecol 2007;29(1):38-43.

8. Brown KL, RidoutDA, Hoskote A, Verhulst L, et al. Delayed diagnosis of congenital heart disease worsens preoperative condition and outcome of surgery in neonates. Heart 2006;92(9):1298-302.

9. SatomiG, YasukochiS, Shimizu T, TakigikuK, etal. Has fetal echocardiography improved the prognosis of congenital heart disease? Comparison of patients with hypoplastic left heart syndrome with and without prenatal diagnosis. Pediatr Int 1999;41(6):728-32.

10. Mahle WT, Clancy RR, McGaurn SP, Goin JE, et al. Impact of prenatal diagnosis on survival and early neurologic morbidity in neonates with the hypoplastic left heart syndrome. Pediatrics 2001;107(6):1277-82.

11. Bartlett JM, Wypij D, Bellinger DC, Rappaport LA, et al. Effect of prenatal diagnosis on outcomes in D-transposition of the great arteries. Pediatrics 2004;113(4):e335-40.

12. Kumar RK, Newburger JW, Gauvreau K, Kamenir SA, et al. Comparison of outcome when hypoplastic left heart syndrome and transposition of the great arteries are diagnosed prenatally versus when diagnosis of these two conditions is made only postnatally. Am J Cardiol 1999;83(12):1649-53.

\title{
Implementación de un programa para prevenir el daño neurológico de la encefalopatía hipóxico isquémica en Buenos Aires. Hipotermia terapéutica
}

\author{
Implementation in Buenos Aires City of a program to prevent neurological \\ damage caused by hypoxic-ischemic encephalopathy. Therapeutic hypothermia
}

\author{
Dra. Mariana Valera ${ }^{a}$, Dr. Juan Pablo Berazategui ${ }^{a}$ Dra. Gladys Saa ${ }^{a}$, Dra. Carolina Olmo Herrera ${ }^{a}$, \\ Dra. Teresa Sepúlveda ${ }^{a}$,Dra. María Fernanda Buraschi ${ }^{a}$, Dr. Sebastián Gacio ${ }^{b}$, Lic. Cristina Villalba ${ }^{a}$, \\ Lic. Inés Belosoc, Dra. Graciela Basso ${ }^{a}$, Dr. Waldemar Carlo y Dr. Jorge Tavosnanska ${ }^{a}$
}

a. Unidad de Neonatología.

b. Servicio de Pediatría.

c. Servicio de Fonoaudiología.

Hospital General de Agudos "Juan A. Fernández"

(HGAJAF), Ciudad Autónoma de Buenos Aires,

República Argentina.

d. División de Neonatología, Universidad de Alabama, EE.UU.

\section{Correspondencia:}

Dr. Jorge Tavosnanska, jtavos@intramed.net

Financiamiento: Ninguno.

Conflicto de intereses: El Servicio de Neonatología del Hospital General de Agudos "Juan A. Fernández" recibió en calidad de préstamo los equipos Cool Cap ${ }^{\circledR}$ de la empresa Medix ${ }^{\circledR}$ by Natus ${ }^{\circledR}$ para hipotermia selectiva y Amrra-Term ${ }^{\circledR}$ para hipotermia corporal total, además del equipo CFM Olympic $6000 \AA$ de la empresa Medix $®$ by Natus $₫$ para el monitoreo de la función cerebral.

\section{RESUMEN}

La hipotermia terapéutica es el estándar de cuidado para la encefalopatía hipóxica isquémica (EHI). Se regionalizó este tratamiento en la red perinatal de la Ciudad de Buenos Aires. Los objetivos de esta presentación son los siguientes: 1. Describir la implementación del programa de hipotermia en la red; 2. Reportar la mortalidad, complicaciones y efectos adversos asociados al tratamiento.

El programa se implementó en etapas: 1) 2009-2010. Capacitación y entrenamiento en el uso del equipamiento. 2) 2010-2014. Tratamiento y seguimiento de los pacientes con EHI moderada o grave. Hasta octubre de 2014, 27 neonatos recibieron hipotermia terapéutica con EHI moderada $(n=15)$ y grave $(n=12)$. Ninguno falleció durante el tratamiento. Se perdieron 3 neonatos en el seguimiento. Delos 16 sobrevivientes mayores de un año, 3 tienen discapacidad neurológica grave. Fue factible implementar el programa. Es prioritario capacitar para la identificación de pacientes con EHI.

Palabras clave: recién nacido, encefalopatía hipóxica isquémica, hipotermia terapéutica.

http:/ /dx.doi.org/10.5546/aap.2015.437 


\section{INTRODUCCIÓN}

La hipotermia terapéutica (HT) para la encefalopatía hipóxico isquémica (EHI) neonatal es el estándar de cuidado en el mundo desarrollado.

Varios ensayos clínicos demuestran que la reducción de la temperatura cerebral en $3-4{ }^{\circ} \mathrm{C}$, mediante hipotermia corporal total o selectiva, constituye una intervención eficaz y segura para reducir la mortalidad y la discapacidad mayor en los sobrevivientes..$^{1-3}$ Este enfriamiento es iniciado antes de las 6 horas de vida y mantenido durante 72 horas, tras lo cual se realiza un recalentamiento lento. La hipotermia parece frenar diversas cascadas bioquímicas que, durante esta fase, conducen al daño: reducción del metabolismo cerebral (fallo energético secundario), supresión de la muerte celular apoptótica, de la reacción inflamatoria y de la activación microglial. ${ }^{4-8}$ Realzan su potencial utilidad clínica la prolongación de la duración de la ventana terapéutica y el efecto sinérgico con otras estrategias neuroprotectoras. ${ }^{9}$

La eficacia y seguridad observada en los ensayos clínicos ha sido ratificada en, al menos, cuatro metaanálisis (Edwards et al. ${ }^{9}$, Schulzke et al. ${ }^{10}$, Shah et al. ${ }^{11}$ y Jacobs et al. ${ }^{12}$ ).

Proporcionar esta intervención en la red perinatal de la Ciudad de Buenos Aires con una tasa anual de 30000 nacimientos requiere una coordinación eficaz dentro del sistema de salud. Nosotros decidimos poner en marcha un programa para ofrecer hipotermia terapéutica a los recién nacidos ( $\mathrm{RN})$ con EHI que nacieran dentro de la red. Como en otras regiones del mundo, planteamos esta estrategia dentro de un sistema perinatal regionalizado. Para ello se estableció un convenio y criterios de urgencia con el Sistema de Atención Médica de Emergencias (SAME) de la Ciudad de Buenos Aires, SAME neonatal, para la correcta derivación y llegada oportuna del paciente.

\section{OBJETIVOS}

1. Describir el proceso de implementación del programa de hipotermia terapéutica en la red perinatal de la Ciudad Autónoma de Buenos Aires (CABA).

2. Reportar la mortalidad, complicaciones y efectos adversos asociados al tratamiento.

\section{EL PROTOCOLO}

La implementación se realizó en dos etapas.

Primera etapa (junio de 2009-junio de 2010):
Diseño de la estrategia para la implementación de la terapéutica.

1. Formación de un grupo de trabajo.

2. Elección del protocolo por implementar.

3. Revisión de todos los pasos de la atención neonatal desde la sala de recepción hasta la Unidad de Cuidados Intensivos Neonatales (UCIN) para asegurar el cumplimiento de las normativas vigentes al año 2011 del Programa de Reanimación Neonatal de la Sociedad Argentina de Pediatría, para lo cual se certificó al 100\% del plantel en dichas normas.

4. Capacitación a todos los profesionales de la Unidad, realizando, en los diferentes turnos del plantel de enfermería y de médicos, entrenamientos en la implementación del "nuevo" protocolo (simulación de casos clínicos, uso de los equipos de hipotermia y monitorio de la función cerebral, controles por realizar con la confección de las planillas correspondientes).

5. Capacitación a todos los jefes de los servicios de Neonatología de la mencionada red sobre el funcionamiento del programa.

6. Se realizaron charlas informativas en las 12 maternidades de la red sobre el programa, criterios de inclusión, vías de comunicación, activación del sistema de traslado neonatal SAME.

7. Establecimiento de un código de hipotermia por parte del SAME para la correcta derivación y llegada oportuna del paciente.

8. Acuerdo con el SAME sobre la forma de enfriamiento y los controles por realizar durante el traslado para el correcto mantenimiento de la temperatura central, para evitar oscilaciones.

9. Incorporación al personal de la Unidad de Neonatología de un neurólogo infantil como parte del grupo de trabajo y seguimiento.

10. Establecimiento de una normativa para el seguimiento de los pacientes incluidos.

Segunda etapa (julio de 2010-octubre de 2014): Implementación del protocolo de hipotermia.

1. Puesta en marcha de la terapéutica.

2. Creación de una base de datos.

3. Desarrollo de un programa de seguimiento específico para evaluar los resultados neuromadurativos (escala Bayley III).

4. Reuniones del grupo de trabajo para reevaluar el programa, cumplimiento de objetivos y mejoras de calidad (auditoría interna de los procesos). 
5. Incorporación de equipamiento nuevo (luego de la autorización por parte de la Administración Nacional de Medicamentos, Alimentos y Tecnología Médica -ANMAT-, agregamos el equipo nacional Amrra-Term ${ }^{\circledR}$ para realizar hipotermia corporal total, además de seguir realizando hipotermia selectiva con el equipo $\left.\mathrm{Cool} \mathrm{Cap}^{\circledR}\right)$.

6. Revisión de la bibliografía actual sobre nuevas terapéuticas coadyuvantes.

\section{Criterios de inclusión para}

el ingreso al protocolo

1. RN con edad gestacional (EG) $\geq 36$ semanas y peso al nacer $(\mathrm{PN}) \geq 1800$ gramos con $\mathrm{EHI}$ moderada o grave (Sarnat \& Sarnat).

2. Puntaje de Apgar $\leq 5$ a los 10 minutos.

3. $\mathrm{pH}$ de cordón $\mathrm{y} / \mathrm{o} 1^{\text {era }}$ hora de vida $\leq 7 \mathrm{y} / \mathrm{o}$ déficit de bases $\geq-16$.

4. Presencia de evento hipóxico centinela (por ejemplo, desprendimiento de placenta).

5. Necesidad de asistencia ventilatoria $\geq 10$ minutos.

6. En el caso de ser trasladado a nuestro centro, se solicitó la firma del consentimiento informado por parte de los padres para el ingreso al protocolo.

7. Poder arribar, tras la solicitud de derivación, al Hospital Fernández antes de las $6 \mathrm{~h}$ posteriores al nacimiento con control continuo de temperatura central. En caso de haber comenzado el tratamiento activo sin equipamiento adecuado, se valoró la posibilidad del ingreso entre las 6 y las 12 horas de vida.

\section{Criterios de exclusión}

1. Paciente con encefalopatía leve.

2. Pacientes que por su extrema gravedad no pudieran ser trasladados (evaluados en forma conjunta por un médico del SAME).

3. No poder arribar a la UCIN antes de las $6 \mathrm{~h}$ posteriores al nacimiento sin tratamiento activo.

\section{Experiencia clínica}

Debido a la baja prevalencia de esta patología y a su mayor incidencia en centros de mediana y baja complejidad, nosotros estimamos que más de la mitad de los niños elegibles serían derivados (Figura 1). Si bien la cantidad total fue mucho menor en el período descrito, la proporción entre propios y referenciados fue la esperada. Los pacientes fueron transferidos con la firma previa del consentimiento informado, pero desconocemos cuántos padres rechazaron la firma para ser referenciados a centros de nivel III. El motivo del rechazo por parte de nuestra Unidad fue no haber cumplido con los criterios de inclusión y fueron aceptadas el 93\% de las solicitudes de derivación.

Respecto al tratamiento ofrecido a los pacientes con hipotermia corporal total (HCT) o hipotermia selectiva (HS), cabe aclarar que el

FIGURA 1. Diagrama de selección y seguimiento de los pacientes que recibieron hipotermia terapéutica

Total de pacientes con criterios para ingresar a hipotermia: $n=29$

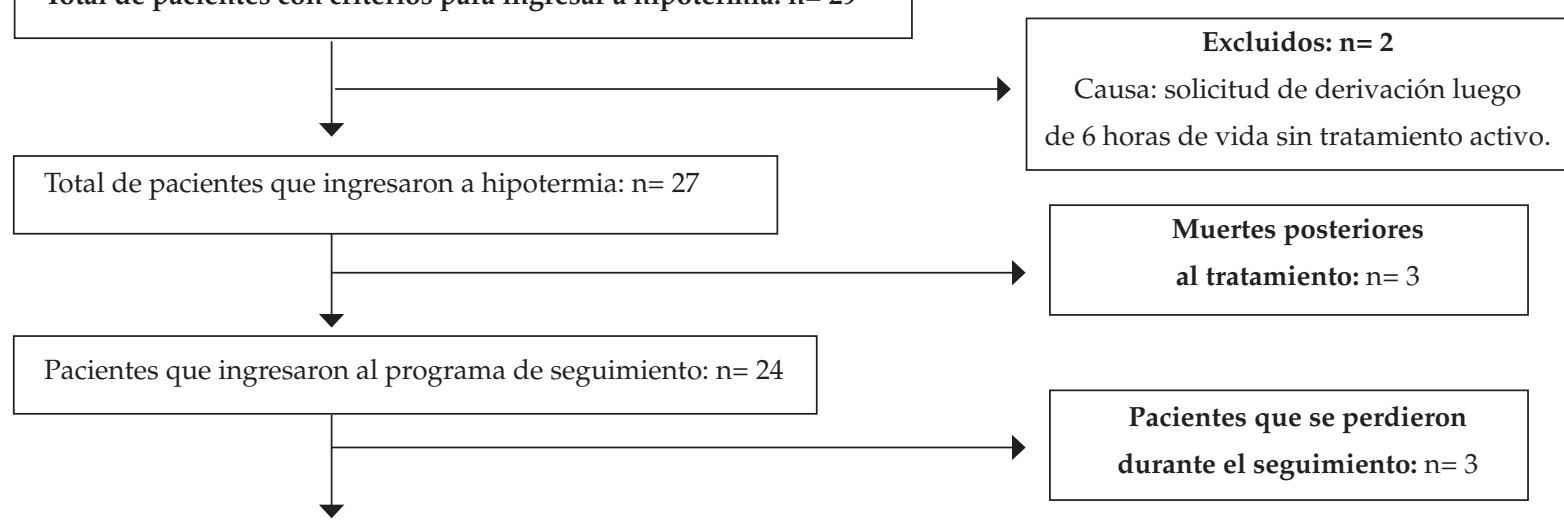

Pacientes en seguimiento: $n=21$ 
equipo de HS fue el primero en ser autorizado por la ANMAT para ser utilizado como tratamiento de la EHI y, por este motivo, los primeros pacientes fueron tratados solo con este equipo. Luego fueron aprobados dos equipos de HCT, uno de origen extranjero y uno de fabricación nacional, ambos servocontrolados. Debido a que, en las revisiones sistemáticas publicadas hasta el día de la fecha, no se ha encontrado una diferencia estadísticamente significativa en cuanto al resultado principal de los estudios comparados, actualmente, ofrecemos uno u otro equipamiento según la disponibilidad..$^{11}$ No hemos tenido complicaciones ni tampoco diferencias en los resultados obtenidos con el uso de ambos equipos junto con el monitoreo de la función cerebral con el equipo CFM Olympic $6000^{\circledR}$.

La frecuencia de los eventos centinelas y complicaciones asociadas al tratamiento fueron similares a las reportadas internacionalmente. No

TABLA 1. Características de los pacientes que ingresaron al protocolo de hipotermia terapéutica

\begin{tabular}{|c|c|c|}
\hline Variables perinatales y de reanimación & $\begin{array}{c}\mathrm{n}=27 \\
\text { Media (DE) }\end{array}$ & n (\%) \\
\hline Peso & $3281,11 \mathrm{~g}(724,4)$ & \\
\hline Edad gestacional & 39,03 sem. $(1,5)$ & \\
\hline Vaginal & & $15(55,5)$ \\
\hline Fórceps & & $1(3,7)$ \\
\hline Cesárea & & $11(40,7)$ \\
\hline Historia perinatal - bradicardia fetal & & $11(40,7)$ \\
\hline Historia perinatal - expulsivo prolongado & & $8(29,6)$ \\
\hline Historia perinatal - desprendimiento de placenta & & $4(14,8)$ \\
\hline Historia perinatal - taquicardia fetal & & $1(3,7)$ \\
\hline Historia perinatal - procidencia de cordón & & $2(7,4)$ \\
\hline Historia perinatal - circular de cordón & & $2(7,4)$ \\
\hline Líquido amniótico meconial & & $9(33,3)$ \\
\hline Podálica & & $1(7,1)$ \\
\hline Apgar $1^{\mathrm{er}}$ minuto $\leq 5$ & & $14(100)$ \\
\hline Apgar $5^{\circ}$ minuto $\leq 5$ & & $8(57,1)$ \\
\hline Solo VPP & & $9(33,3)$ \\
\hline $\mathrm{VPP}+\mathrm{TET}$ & & $7(25,9)$ \\
\hline $\mathrm{VPP}+\mathrm{TET}+$ masaje cardíaco & & $7(25,9)$ \\
\hline $\mathrm{VPP}+\mathrm{TET}+$ masaje cardíaco + drogas & & $4(14,8)$ \\
\hline Hipotermia selectiva & & $21(77,7)$ \\
\hline Hipotermia corporal total & & $6(22,2)$ \\
\hline Hora de ingreso a la hipotermia (horas) & $4,07(1,9)$ & \\
\hline Temperatura de ingreso & $34,65(0,7)$ & \\
\hline Ph de cordón o en primera hora de vida & $6,88(0,2)$ & \\
\hline Exceso de base & $-18,3(5,4)$ & \\
\hline
\end{tabular}

VPP: ventilación a presión positiva; TET: tubo endotraqueal.

TABLA 2. Complicaciones asociadas durante la hipotermia terapéutica

\begin{tabular}{lc}
\hline Complicaciones asociadas a la hipotermia & $\mathbf{n = 2 7 ( \% )}$ \\
\hline Ninguna & $12(44,44)$ \\
Bradicardia & $2(7,40)$ \\
Hipotensión & $2(7,40)$ \\
Alteración del coagulograma & $10(37,03)$ \\
Plaquetopenia & $4(14,81)$ \\
\hline
\end{tabular}


murió ningún niño durante el tratamiento o por complicaciones relacionadas con este (Tablas 1 y $2)$, pero $3(11 \%)$ murieron antes del mes de vida.

Se realizó una resonancia magnética de cerebro en 20 de 27 pacientes entre los 5 y los 20 días de vida, y 6 fueron patológicas.

Se perdieron 3 pacientes durante el seguimiento y 5 aún no tienen 1 año de edad. De los 16 sobrevivientes mayores de 1 año, 3 tienen discapacidad neurológica grave y 1 , síndrome genético en estudio (Figura 2).

\section{COMENTARIO}

La posibilidad actual de prevenir o aminorar el daño cerebral asociado a la EHI mediante la hipotermia moderada sostenida ha conducido a establecer programas asistenciales específicos.

De acuerdo con nuestra experiencia, fue factible implementar un programa de hipotermia terapéutica. La mortalidad, morbilidad y complicaciones asociadas a este tratamiento fueron similares a las publicadas internacionalmente.

Debido a que la eficacia terapéutica de la hipotermia parece estar relacionada con el inicio antes de las primeras 6 horas de vida, se requiere: a) centralizar los programas en varios centros terciarios por área geográfica; y b) establecer un "código hipotermia" que asegure el traslado urgente y seguro del paciente desde el centro emisor a un centro de referencia. Para que este código sea útil, es esencial la adecuada coordinación operativa entre todos los agentes implicados. ${ }^{13}$

Si bien aún no contamos, tanto a nivel nacional como en la red de la CABA, con tasas certeras de la prevalencia de niños que nacen con EHI, estimamos que la cantidad de niños derivados para realizar un tratamiento neuroprotector fue mucho menor que la esperada, por lo cual consideramos que deberíamos implementar una herramienta para poder capacitar y tener mayor impacto en las maternidades de la red perinatal de la CABA con el fin de aumentar las derivaciones y lograr la equidad en el tratamiento.

\section{REFERENCIAS}

1. Gluckman PD, Wyatt JS, Azzopardi D, Ballard R, et al. Selective head cooling with mild systemic hypothermia after neonatal encephalopathy: multicentre randomised trial. Lancet 2005;365(9460):663-70.

2. Shankaran S, Laptook AR, Ehrenkranz RA, Tyson JE, et al. Whole-body hypothermia for neonates with hypoxic-ischemic encephalopathy. New Engl J Med 2005;353(15):1574-84.

3. Azzopardi DV, Strohm B, Edwards AD, Dyet L, et al. Moderate hypothermia to treat perinatal asphyxial encephalopathy. N Engl J Med 2009;361(14):1349-58.

4. Ellis M, Manandhar D. Progress in perinatal asphyxia. Semin Neonatol 1999;4(3):183-91.

5. Perlman JM. Interventions strategies for neonatal hypoxicischemic cerebral injury. Clin Ther 2006;28(9):1353-65.

6. Gunn AJ, Gunn TR, Gunning MI, Williams CE, et al. Neuroprotection with prolonged head cooling started before postischemic seizures in fetal sheep. Pediatrics 1998;102(5):1098-106.

$\mathrm{n}=13$

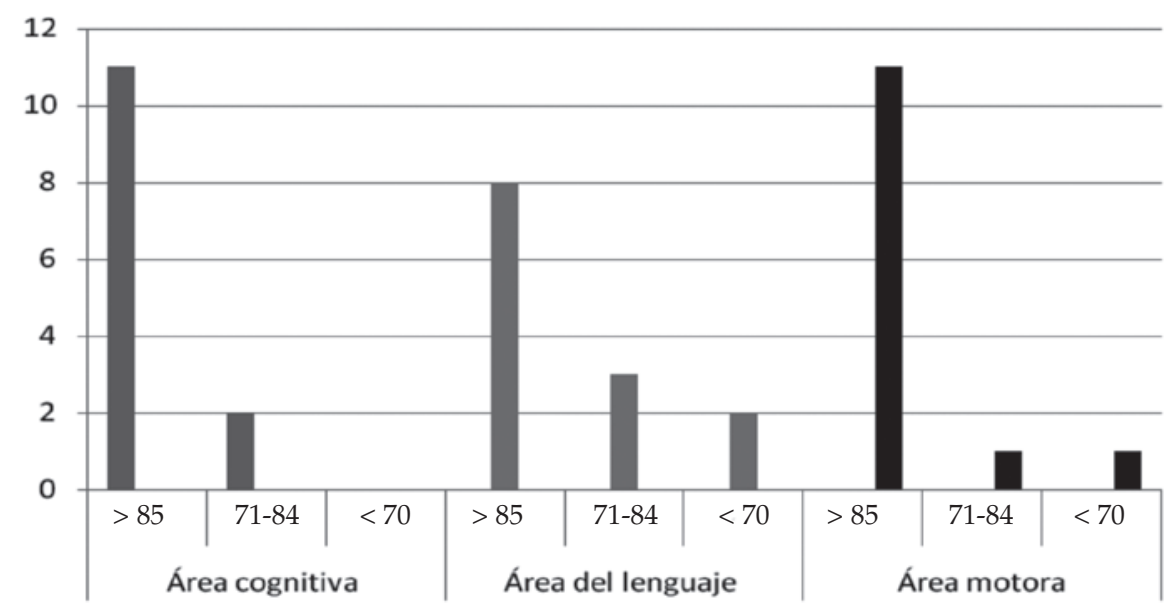


7. Thoresen M, Wyatt J. Keeping a cool head, posthypoxic hypothermia-an old idea revisited. Acta Paediatr 1997;86(10):1019-33.

8. Gunn AJ. Cerebral hypothermia for prevention of brain injury following perinatal asphyxia. Curr Opin Pediatr 2000;12(2):111-5.

9. Edwards AD, Azzopardi DV. Therapeutic hypothermia following perinatal asphyxia. Arch Dis Child Fetal Neonatal Ed 2006;91(2):F127-31.

10. Schulzke SM, Rao S, Patole SK. A systematic review of cooling for neuroprotection in neonates with hypoxic ischemic encephalopathy- are we there yet? BMC Pediatr 2007;7:30.
11. Shah PS, Ohlsson A, Perlman M. Hypothermia to treat neonatal hypoxic ischemic encephalopathy: systematic review. Arch Pediatr Adolesc Med 2007;161(10):951-8.

12. Jacobs S, Hunt R, Tarnow-Mordi W, Inder T, et al. Cooling for newborns with hypoxic ischaemic encephalopathy. Cochrane Database Syst Rev 2007;(4):CD00311.

13. García-Alix A, González de Dios J. La encefalopatía hipóxico-isquémica en el recién nacido a término ha dejado de ser una entidad huérfana. Implicaciones para la práctica y necesidad de un "código hipotermia". Evid Pediatr 2010;6(2):27.

Ser humilde para con los superiores es un deber; para con los iguales, una muestra de cortesía; para con los inferiores, una prueba de nobleza. 